“C 2016 IEEE. Personal use of this material is permitted. Permission from IEEE must be obtained for all other uses, in any current or future media, including reprinting/republishing this material for advertising or promotional purposes, creating new collective works, for resale or redistribution to servers or lists, or reuse of any copyrighted component of this work in other works." 


\title{
DNA-GA: A New Approach of Network Performance Analysis
}

\author{
Ming Ding ${ }^{\ddagger}$, David López Pérez ${ }^{\dagger}$, Guoqiang Mao $\$ \ddagger \rrbracket \S$, Zihuai Lin \\ ${ }^{\ddagger}$ Data61, Australia, ${ }^{\dagger}$ Bell Labs, Nokia, Ireland \\ $\nVdash$ School of Computing and Communication, University of Technology Sydney, Australia \\ $\sqcap$ School of Electronic Information \& Communications, Huazhong University of Science \& Technology, Wuhan, China \\ $\S$ School of Information and Communication Engineering, Beijing University of Posts and Telecommunications, Beijing, China \\ The University of Sydney, Australia
}

\begin{abstract}
In this paper, we propose a new approach of network performance analysis, which is based on our previous works on the deterministic network analysis using the Gaussian approximation (DNA-GA). First, we extend our previous works to a signal-to-interference ratio (SIR) analysis, which makes our DNA-GA analysis a formal microscopic analysis tool. Second, we show two approaches for upgrading the DNA-GA analysis to a macroscopic analysis tool. Finally, we perform a comparison between the proposed DNA-GA analysis and the existing macroscopic analysis based on stochastic geometry. Our results show that the DNA-GA analysis possesses a few special features: (i) shadow fading is naturally considered in the DNAGA analysis; (ii) the DNA-GA analysis can handle non-uniform user distributions and any type of multi-path fading; (iii) the shape and/or the size of cell coverage areas in the DNA-GA analysis can be made arbitrary for the treatment of hotspot network scenarios. Thus, DNA-GA analysis is very useful for the network performance analysis of the 5 th generation $(5 \mathrm{G})$ systems with general cell deployment and user distribution, both on a microscopic level and on a macroscopic level.
\end{abstract}

\section{INTRODUCTION}

Due to their potential for large performance gains, dense orthogonal deployments of small cell networks ( $\mathrm{SCNs}$ ) within the existing macrocell networks ${ }^{1}$ gained much momentum in the design of the 4th generation (4G) systems [1], and are envisaged as the workhorse for capacity enhancement in the 5 th generation $(5 \mathrm{G})$ systems [2]. In this context, new and more powerful network performance analysis tools are needed.

Network performance analysis tools can be broadly classified into two groups, i.e., macroscopic analysis [3,4] and microscopic analysis [5-10]. The macroscopic analysis usually assumes that user equipments (UEs) and/or base stations (BSs) are randomly deployed, often following a homogeneous Poisson distribution to invoke the stochastic geometry theory $[3,4]$. In essence, the macroscopic analysis investigates network performance at a high level, such as coverage probability and signal-to-interference ratio (SIR) distribution, by averaging over all possible UE and BS deployments $[3,4]$. Instead, the

\footnotetext{
${ }^{1}$ Orthogonal deployment means that small cells and macrocells operating on different frequency spectrum, i.e., Small Cell Scenario \#2a defined in [1] \& Guoqiang Mao's research is supported by Australian Research Council (ARC) Discovery projects DP110100538 and DP120102030 and Chinese National Science Foundation project 61428102.
}

microscopic analysis allows for a more detailed analysis and is often conducted assuming that UEs are randomly placed but that BS locations are known [5-10].

Within the microscopic analysis and paying special attention to uplink (UL), in [5], the authors considered a single UL interfering cell with a disk-shaped coverage area and presented closed-form expressions for the UL interference considering both path loss and shadow fading. In [6], the authors conjectured that the UL interference in a hexagonal grid based cellular network may follow a lognormal distribution, which was verified via simulation. In [7] and [8], we went a step further and analytically derived an upper bound of the error in approximating the dB-scale UL interference from a single cell by a Gaussian distribution. Such error was measured by the Kolmogorov-Smirnov (KS) distance [11] between the real cumulative density function (CDF) and the approximate CDF, and it was shown to be small for practical SCNs. On the basis of this single-cell interference analysis, we further investigated the approximate distribution of the aggregate UL interference in a multi-cell scenario as a power lognormal distribution. For more practical networks, in [9] and [10], we also investigated the network performance of SCNs in current $4 \mathrm{G}$ networks using system-level simulations.

In this paper, our objective is to extend our previous works in [7] and [8] to analyze the UL SIR performance, and create a novel and compelling approach for network performance analysis that can unify the macroscopic and the microscopic analyses within a single framework. To that end, our work is composed of the following three steps:

1) The extension of the UL interference analysis in [7] and [8] to the UL SIR analysis, which makes our analysis a formal microscopic analysis tool.

2) The upgrade of the developed microscopic analysis tool to a macroscopic analysis tool.

3) The comparison between the proposed macroscopic analysis tool and the existing macroscopic analysis based on stochastic geometry.

Since the macroscopic and the microscopic analyses are unified in our framework based on a deterministic network analysis (DNA) using the Gaussian approximation (GA) pre- 
sented in [7] and [8], our framework will be referred to as the DNA-GA analysis hereafter. Our main contributions are:

1) Based on the GA theorem presented in [7] and [8], the approximate distributions of the UL signal power and the UL SIR for the interested UE are derived in tractable expressions using the Gauss-Hermite numerical integration [12], giving rise to the DNA-GA analysis.

2) Although the DNA-GA analysis stands alone as a solid contribution to the family of microscopic analysis, two approaches for upgrading the DNA-GA analysis to a macroscopic analysis are further investigated. The first one is the semi-analytical approach, which directly averages the performance given by many DNA-GA analyses over many random BS deployments to obtain the performance of the macroscopic analysis. The second one is the analytical approach, which constructs an idealistic and deterministic BS deployment, and then conducts the DNA-GA analysis on such BS deployment to obtain an upper-bound performance of the macroscopic analysis.

3) Interesting results on the comparison between the DNAGA analysis and the stochastic geometry analysis [4] are presented. Our results show that the DNA-GA analysis qualifies as a new network performance analysis tool with a few special merits over stochastic geometry: (i) Shadow fading is naturally considered in the DNA-GA analysis, while stochastic geometry usually cannot; (ii) Non-uniform UE distributions and any type of multipath fading can be treated in the DNA-GA analysis, while stochastic geometry usually cannot; (iii) Apart from the common assumption on the cell coverage areas as Voronoi cells made by stochastic geometry, the shape and/or the size of cell coverage areas in the DNA-GA analysis can be made arbitrary, making it suitable for the network performance analysis of hotspot SCNs.

\section{Network Scenario And System Model}

In this paper, we consider UL transmissions, and assume that each small cell BS only schedules one UE in each frequency/time resource, i.e., resource block $(\mathrm{RB})$. This is a reasonable assumption in line with $4 \mathrm{G}$ networks, i.e., Long Term Evolution (LTE) [1] and Worldwide Interoperability for Microwave Access (WiMAX) [13]. Note that small cell BSs serving no UE do not contribute to the UL interference, thereby those BSs are ignored in the analysis.

Regarding the network scenario, we consider a SCN with multiple small cells operating on the same carrier frequency, as shown in Fig. 1. In more detail, the SCN consists of $B$ small cells, each of which is managed by a BS. The network includes the small cell of interest denoted by $C_{1}$ and $B-1$ interfering small cells denoted by $C_{b}, b \in\{2, \ldots, B\}$. We focus on a particular RB, and denote by $K_{b}$ the active UE associated with small cell $C_{b}$ in such RB. Moreover, we denote by $R_{b}$ the coverage area of small cell $C_{b}$, in which its associated UEs are randomly distributed. Note that the coverage areas of adjacent small cells may overlap due to the arbitrary shapes and sizes of $\left\{R_{b}\right\}, b \in\{2, \ldots, B\}$.

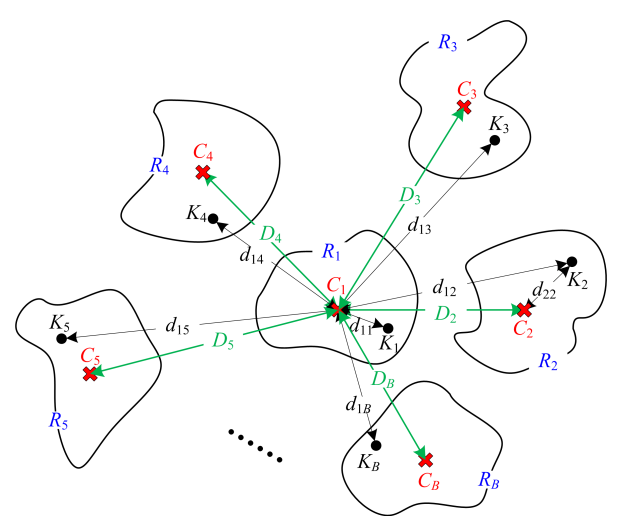

Fig. 1. A schematic model of the considered SCN.

The distance (in $\mathrm{km}$ ) from the $\mathrm{BS}$ of $C_{b}$ to the $\mathrm{BS}$ of $C_{1}, b \in\{1, \ldots, B\}$, and the distance from UE $K_{b}$ to the BS of $C_{m}, b, m \in\{1, \ldots, B\}$, are denoted by $D_{b}$ and $d_{b m}$, respectively. Since DNA-GA is a microscopic analysis tool, we consider a deterministic deployment of BSs, i.e., the set $\left\{D_{b}\right\}$ is known, while each UE $K_{b}$ is randomly distributed in $R_{b}$ with a distribution function $f_{Z_{b}}(z), z \in R_{b}$. Hence, $d_{b m}$ is a random variable (RV), whose distribution cannot be readily expressed in an analytical form due to the arbitrary shape and size of $R_{b}$, and the arbitrary form of $f_{Z_{b}}(z)$. Regarding $R_{b}$ and $f_{Z_{b}}(z)$, we have two remarks in the following.

Remark 1: Unlike the existing works, e.g., [3-6, 9-10], where only the uniform UE distribution was considered, DNA-GA can handle any probability density function (PDF) of general UE distribution, here denoted by $f_{Z_{b}}(z)$, where $0<f_{Z_{b}}(z)<$ $+\infty, z \in R_{b}$ and its integral over $R_{b}$ equals to one, i.e., $\int_{R_{b}} f_{Z_{b}}(z) d z=1$.

Remark 2: Even if $f_{Z_{b}}(z)$ is constant with $z$, we can only say that the UE distribution is uniform within the small cell coverage area $R_{b}$, but we cannot guarantee that the UE distribution is uniform within the entire scenario, because no UEs are deployed outside the hotspot areas $\left\{R_{b}\right\}$, which may cause the non-uniformity of UE distribution within the entire scenario. Instead, in stochastic geometry [3,4], UEs are usually assumed to be uniformly distributed within the entire scenario, creating Voronoi cells, which is less general and practical than our assumption of $R_{b}$ and $f_{Z_{b}}(z)$. Note that in the sequel, the characterization of UE distribution is meant within $R_{b}$.

Based on the definition of $d_{b m}$, the path loss in $\mathrm{dB}$ from UE $K_{b}$ to the BS of $C_{m}$ is modeled as

$$
L_{b m}=A+\alpha \times 10 \log _{10} d_{b m},
$$

where $A$ is the path loss in $\mathrm{dB}$ at the reference distance of $d_{b m}=1$ and $\alpha$ is the path loss exponent. In practice, $A$ and $\alpha$ are constants obtainable from field tests [14]. Note that $L_{b m}$ is a $\mathrm{RV}$ due to the randomness of $d_{b m}$.

The shadow fading in $\mathrm{dB}$ from $\mathrm{UE} K_{b}$ to the $\mathrm{BS}$ of $C_{m}$ is denoted by $S_{b m}$, and is usually assumed to follow a lognormal distribution [14]. Based on this assumption, $S_{b m}$ is modeled as an independently and identically distributed (i.i.d.) zero-mean Gaussian RV with a variance of $\sigma_{\text {Shad }}^{2}$, i.e., $S_{b m} \sim \mathcal{N}\left(0, \sigma_{\text {Shad }}^{2}\right)$. 
The UL transmission power in $\mathrm{dBm}$ of $\mathrm{UE} K_{b}$ is denoted by $P_{b}$, and is subject to a semi-static power control (PC) mechanism, e.g., the fractional path loss compensation (FPC) scheme [14]. Based on this FPC scheme, $P_{b}$ is modeled as

$$
P_{b}=P_{0}+\eta\left(L_{b b}+S_{b b}\right),
$$

where $P_{0}$ is the target received power in $\mathrm{dBm}$ on the considered RB at the BS, $\eta \in(0,1]$ is the FPC factor, and $L_{b b}$ and $S_{b b} \sim \mathcal{N}\left(0, \sigma_{\text {Shad }}^{2}\right)$ have been discussed above.

The multi-path fading channel from UE $K_{b}$ to the BS of $C_{m}$ is denoted by $\mathbf{h}_{b m} \in \mathbb{C}$, where we assume that each UE and each BS are equipped with one omni-directional antenna. It is important to note that we consider a general type of multipath fading by assuming that the effective channel gain in $\mathrm{dB}$ associated with $\mathbf{h}_{b m}$ is defined as $H_{b m}=10 \log _{10}\left|\mathbf{h}_{b m}\right|^{2}$, which follows an i.i.d. distribution with a PDF of $f_{H}(h)$. For example, $\left|\mathbf{h}_{b m}\right|^{2}$ can be characterized by an exponential distribution or a Gamma distribution in case of Rayleigh fading or Nakagami fading, respectively [15]. And hence, the distribution of $H_{b m}$ can be derived analytically.

Finally, we ignore the additive noise because the $4 \mathrm{G}$ and the $5 \mathrm{G}$ SCNs generally work in the interference-limited region [2].

\section{The Proposed DNA-GA ANALYSis}

The proposed DNA-GA analysis consists of three steps, i.e., the interference analysis, the signal power analysis, and the SIR analysis, which are presented in the following.

\section{A. The Interference Analysis}

Based on the definition of RVs discussed in Section II, the $U L$ received interference power in $\mathrm{dBm}$ from $\mathrm{UE} K_{b}$ to the BS of $C_{1}$ can be written as

$$
\begin{aligned}
I_{b} & \stackrel{(a)}{=} P_{b}-L_{b 1}-S_{b 1}+H_{b 1} \\
& =P_{0}+\left(\eta L_{b b}-L_{b 1}\right)+\left(\eta S_{b b}-S_{b 1}\right)+H_{b 1} \\
& \triangleq\left(P_{0}+L+S\right)+H_{b 1}, \\
& \triangleq I_{b}^{(1)}+H_{b 1},
\end{aligned}
$$

where (2) is plugged into the step (a) of (3), and $L$ and $S$ are defined as $L \triangleq\left(\eta L_{b b}-L_{b 1}\right)$ and $S \triangleq\left(\eta S_{b b}-S_{b 1}\right)$, respectively. Apparently, $L$ and $S$ are independent RVs. Besides, the first part of $I_{b}$ is further defined as $I_{b}^{(1)} \triangleq\left(P_{0}+L+S\right)$. Since $S_{b b}$ and $S_{b 1}(b \in\{2, \ldots, B\})$ are i.i.d. zero-mean Gaussian $\mathrm{RVs}$, it is easy to show that $S$ is also a Gaussian RV, whose mean and variance are

$$
\left\{\begin{array}{l}
\mu_{S}=0 \\
\sigma_{S}^{2}=\left(1+\eta^{2}\right) \sigma_{\text {Shad }}^{2}
\end{array}\right.
$$

From the definition of $I_{b}$ in (3), the aggregate interference power in $\mathrm{mW}$ from all interfering UEs to the $\mathrm{BS}$ of $C_{1}$ can be formulated as

$$
I^{\mathrm{mW}}=\sum_{b=2}^{B} 10^{\frac{1}{10} I_{b}} .
$$

In our previous work [8], we show that the distribution of $I^{\mathrm{mW}}$ can be well approximated by a power lognormal distribution. This approximation is summarized in the following.
1) The Distribution of $I_{b}^{(1)}$ in (3): First, we analyze the distribution of $I_{b}^{(1)}$ shown in (3). Considering a small approximation error, upper-bounded by the KS distance [11] provided in [8], we approximate $I_{b}^{(1)}$ by a Gaussian RV $G_{b}$, whose mean and variance are

$$
\left\{\begin{array}{l}
\mu_{G_{b}}=P_{0}+\mu_{L}+\mu_{S} \\
\sigma_{G_{b}}^{2}=\sigma_{L}^{2}+\sigma_{S}^{2}
\end{array},\right.
$$

where $\mu_{L}$ and $\sigma_{L}^{2}$ are respectively the mean and the variance of $L$, which can be obtained using numerical integration involving $f_{Z_{b}}(z)$ and $R_{b}$ [7,8]. Details are omitted for brevity.

2) The Distribution of $I_{b}$ in (3): Second, we analyze the distribution of $I_{b}=I_{b}^{(1)}+H_{b 1}$ shown in (3). Considering a small approximation error, upper-bounded by the KS distance [11] provided in [8], we approximate $I_{b}$ by another Gaussian RV $Q_{b}$, whose mean and variance are

$$
\left\{\begin{array}{l}
\mu_{Q_{b}}=\mu_{G_{b}}+\mu_{H_{b 1}} \\
\sigma_{Q_{b}}^{2}=\sigma_{G_{b}}^{2}+\sigma_{H_{b 1}}^{2}
\end{array} .\right.
$$

where $\mu_{H_{b 1}}$ and $\sigma_{H_{b 1}}^{2}$ are respectively the mean and the variance of $H_{b 1}$. We omit the details for brevity.

Note that the upper bound of the total approximation error of the above two steps is obtained from the summation of the individual approximation errors of the two steps in closed-form expressions [8]. And it has been shown in [8] that the total approximation error is small for practical SCNs, without any requirement on (i) the uniformity of UE distribution and/or the type of multi-path fading; and (ii) the shape and/or size of cell coverage areas. Intuitively speaking, the results in [8] show that the larger the variance of the Gaussian RV, i.e., $S$ in (6) or $G_{b}$ in (7), the better the approximation in (6) or in (7), due to the increasing dominance of the Gaussian RV.

3) The Distribution of $I^{m W}$ in (5): Third, we invoke the main results in [17-18], which indicate that the sum of multiple independent lognormal RVs can be well approximated by a power lognormal RV. Accordingly, in our case, since each $I_{b}, b \in\{2, \ldots, B\}$ is approximated by a Gaussian $\mathrm{RV} Q_{b}$, their sum $10^{\frac{1}{10}} Q_{b}$ shown in (5) should be well approximated by a power lognormal RV expressed as $\hat{I}^{\mathrm{mW}}=10^{\frac{1}{10}} Q$, where the PDF and CDF of $Q$ [16] can be written as (8) shown on the top of the next page. In (8), $\Phi(x)$ is the CDF of the standard normal distribution, and the parameters $\lambda, \mu_{Q}$ and $\sigma_{Q}$ are obtained from $\left\{\mu_{Q_{b}}\right\}$ and $\left\{\sigma_{Q_{b}}^{2}\right\}$ that are computed by (7). The procedure to obtain $\lambda, \mu_{Q}$ and $\sigma_{Q}$ is omitted here for brevity, but interested readers are referred to Appendix B of [8] for further details. As a result of (8), the PDF and CDF of $\hat{I}^{\mathrm{mW}}$ can be written as (9) shown on the top of the next page, where $\zeta=\frac{10}{\ln 10}$ is a scalar factor originated from the variable change from $10 \log _{10} v$ to $\ln v$.

Finally, we approximate the distribution of $I^{\mathrm{mW}}$ by that of $\hat{I}^{\mathrm{mW}}$ shown in (9) presented on the top of the next page. Note that in this step, the approximation error depends on the approximate error introduced by the power lognormal approximation, which has been shown to be reasonably small and good enough in practical cases [17-18]. 


$$
\begin{gathered}
\left\{\begin{array}{l}
\text { PDF of } Q: f_{Q}(q)=\lambda \Phi^{\lambda-1}\left(\frac{q-\mu_{Q}}{\sigma_{Q}}\right) \frac{1}{\sqrt{2 \pi \sigma_{Q}^{2}}} \exp \left\{-\frac{\left(q-\mu_{Q}\right)^{2}}{2 \sigma_{Q}^{2}}\right\} . \\
\text { CDF of } Q: F_{Q}(q)=\Phi^{\lambda}\left(\frac{q-\mu_{Q}}{\sigma_{Q}}\right)
\end{array}\right. \\
\left\{\begin{array}{l}
\text { PDF of } \hat{I}^{\mathrm{mW}}: f_{\hat{I}^{\mathrm{mW}}}(v)=\lambda \Phi^{\lambda-1}\left(\frac{\zeta \ln v-\mu_{Q}}{\sigma_{Q}}\right) \frac{\zeta}{v \sqrt{2 \pi \sigma_{Q}^{2}}} \exp \left\{-\frac{\left(\zeta \ln v-\mu_{Q}\right)^{2}}{2 \sigma_{Q}^{2}}\right\} \\
\text { CDF of } \hat{I}^{\mathrm{mW}}: F_{\hat{I}^{\mathrm{mW}}}(v)=\Phi^{\lambda}\left(\frac{\zeta \ln v-\mu_{Q}}{\sigma_{Q}}\right)
\end{array}\right.
\end{gathered}
$$

\section{B. The Signal Power Analysis}

The $U L$ received signal power in $\mathrm{dBm}$ from $\mathrm{UE} K_{1}$ to the BS of $C_{1}$ can be written as

$$
\begin{aligned}
X_{1} & \stackrel{(a)}{=} P_{1}-L_{11}-S_{11}+H_{11} \\
& =P_{0}+\left(\eta L_{11}-L_{11}\right)+\left(\eta S_{11}-S_{11}\right)+H_{11} \\
& \triangleq\left(P_{0}+\bar{L}_{11}+\bar{S}_{11}\right)+H_{11}, \\
& \triangleq X_{1}^{(1)}+H_{11},
\end{aligned}
$$

where (2) is plugged into the step (a) of (10). Besides, $\bar{L}_{11}$ and $\bar{S}_{11}$ are defined as $\bar{L}_{11} \triangleq(\eta-1) L_{11}$ and $\bar{S}_{11} \triangleq(\eta-1) S_{11}$, respectively. The first part of $X_{1}$ is further defined as $X_{1}^{(1)} \triangleq$ $P_{0}+\bar{L}_{11}+\bar{S}_{11}$, and it is easy to show that $\bar{S}_{11}$ is a Gaussian $\mathrm{RV}$, whose mean and variance are

$$
\left\{\begin{array}{l}
\mu_{\bar{S}_{11}}=0 \\
\sigma_{\bar{S}_{11}}^{2}=(1-\eta)^{2} \sigma_{\text {Shad }}^{2}
\end{array} .\right.
$$

Similar to the discussion in subsection III-A1, we consider a small approximation error, upper-bounded by the KS distance shown in [8], and we approximate $X_{1}^{(1)}$ by a Gaussian RV $G_{1}$, whose mean and variance are

$$
\left\{\begin{array}{l}
\mu_{G_{1}}=P_{0}+\mu_{\bar{L}_{11}}+\mu_{\bar{S}_{11}} \\
\sigma_{G_{1}}^{2}=\sigma_{\bar{L}_{11}}^{2}+\sigma_{\bar{S}_{11}}^{2}
\end{array},\right.
$$

where $\mu_{\bar{L}_{11}}$ and $\sigma_{\bar{L}_{11}}^{2}$ are respectively the mean and the variance of $\bar{L}_{11}$. As a result, (10) can be re-formulated as

$$
X_{1} \approx G_{1}+H_{11} \triangleq \hat{X}_{1} \text {. }
$$

Note that unlike the discussion in subsection III-A2, it is not accurate to further approximate $\hat{X}_{1}$ by a Gaussian RV, because the randomness of the Gaussian distributed RV $S_{11}$ is largely removed by the UL transmission power control mechanism, rendering a less dominant role of the Gaussian distribution of $G_{1}$ compared with the distribution of $H_{11}$. In other words, $\sigma_{G_{1}}^{2}$ is comparable with or even smaller than the variance of $H_{11}$, making the approximation error large according to our results in [8]. Therefore, we derive the approximate distribution of $X_{1}$ using a different method, presented in Theorem 1 .

Theorem 1. The approximate CDF of $X_{1}$ is derived as

$$
F_{X_{1}}(x) \approx F_{\hat{X}_{1}}(x)=\frac{1}{\sqrt{\pi}} \sum_{m=1}^{M_{0}} w_{m} F_{H_{11}}\left(x-\left(\sqrt{2} \sigma_{G_{1}} a_{m}+\mu_{G_{1}}\right)\right) \text {, }
$$

where $M_{0}$ is the number of terms employed in the GaussHermite numerical integration [12], and the weights $\left\{w_{m}\right\}$ and the abscissas $\left\{a_{m}\right\}$ are tabulated in Table 25.10 of [12].
Proof: Since $G_{1}$ is a Gaussian RV with the mean and the variance shown in (12), the PDF of $G_{1}$ can be written as

$$
f_{G_{1}}(v)=\frac{1}{\sqrt{2 \pi \sigma_{G_{1}}^{2}}} \exp \left\{-\frac{\left(v-\mu_{G_{1}}\right)^{2}}{2 \sigma_{G_{1}}^{2}}\right\} \text {. }
$$

Besides, we assume the CDF of $H_{11}$ to be $F_{H_{11}}(h)$. Hence, the CDF of $X_{1}$ can be approximated by

$$
\begin{aligned}
F_{X_{1}}(x) \approx F_{\hat{X}_{1}}(x) & \\
= & \operatorname{Pr}\left[G_{1}+H_{11} \leq x\right] \\
= & \operatorname{Pr}\left[H_{11} \leq x-G_{1}\right] \\
= & \int_{-\infty}^{+\infty} F_{H_{11}}(x-v) f_{G_{1}}(v) d v \\
& \stackrel{(a)}{=} \int_{-\infty}^{+\infty} F_{H_{11}}(x-v) \frac{1}{\sqrt{2 \pi \sigma_{G_{1}}^{2}}} \exp \left\{-\frac{\left(v-\mu_{G_{1}}\right)^{2}}{2 \sigma_{G_{1}}^{2}}\right\} d v \\
& \stackrel{(b)}{=} \frac{1}{\sqrt{\pi}} \int_{-\infty}^{+\infty} F_{H_{11}}\left(x-\left(\sqrt{2} \sigma_{G_{1}} y+\mu_{G_{1}}\right)\right) \exp \left(-y^{2}\right) d y \\
& \stackrel{(c)}{=} \frac{1}{\sqrt{\pi}} \sum_{m=1}^{M_{0}} w_{m} F_{H_{11}}\left(x-\left(\sqrt{2} \sigma_{G_{1}} a_{m}+\mu_{G_{1}}\right)\right)+R_{M_{0}} \\
\stackrel{(d)}{\approx} & \frac{1}{\sqrt{\pi}} \sum_{m=1}^{M_{0}} w_{m} F_{H_{11}}\left(x-\left(\sqrt{2} \sigma_{G_{1}} a_{m}+\mu_{G_{1}}\right)\right)
\end{aligned}
$$

where the step (a) of (16) is obtained from (15), and the step (b) of (16) is computed using the variable change $v=\sqrt{2} \sigma_{G_{1}} y+\mu_{G_{1}}$. Moreover, the step (c) of (16) is derived using the Gauss-Hermite numerical integration [12], i.e., $\quad \int_{-\infty}^{+\infty} f(y) \exp \left(-y^{2}\right) d y=\sum_{m=1}^{M_{0}} w_{m} f\left(a_{m}\right)+R_{M_{0}}$, where $M_{0}$ is the number of terms in the approximation, the weights $\left\{w_{m}\right\}$ and the abscissas $\left\{a_{m}\right\}$ are tabulated in Table 25.10 of [12] and $R_{M_{0}}$ is a residual error in the order of $\frac{M_{0} !}{2^{M_{0}}\left(2 M_{0}\right) !}$ [12], which decays very fast as $M_{0}$ increases. Finally, the step (d) of (16) is obtained by dropping $R_{M_{0}}$. Our proof is thus completed by comparing (14) and (16).

In case of Rayleigh fading [15], we propose Corollary 2 to compute the approximate expression of $F_{X_{1}}(x)$.

Corollary 2. In case of Rayleigh fading, the approximate CDF of $X_{1}$ can be computed by (14), where

$$
F_{H_{11}}(h)=1-\exp \left(-\exp \left(\frac{h}{\zeta}\right)\right)
$$

where $\zeta=\frac{10}{\ln 10}$. 
Proof: As discussed in Section II, on condition of Rayleigh fading, the channel gain $\left|\mathbf{h}_{11}\right|^{2}$ follows an exponential distribution with unitary mean [15]. Then, our proof is completed by deriving (17) based on the variable change $H_{11}=10 \log _{10}\left|\mathbf{h}_{11}\right|^{2}$. Details are omitted for brevity.

In case of Nakagami fading [15], we propose Corollary 3 to compute the approximate expression of $F_{X_{1}}(x)$.

Corollary 3. In case of Nakagami fading, the approximate CDF of $X_{1}$ can be computed by (14), where

$$
F_{H_{11}}(h)=\frac{1}{\Gamma(k)} \gamma\left(k, \frac{1}{\theta} \exp \left(\frac{h}{\zeta}\right)\right),
$$

where $\Gamma(\cdot)$ and $\gamma(\cdot, \cdot)$ are respectively the gamma and the incomplete gamma functions [12], $k$ and $\theta$ are respectively the shape and the scale parameters of the Gamma distribution associated with the channel gain of Nakagami fading [15].

Proof: As discussed in Section II, on condition of Nakagami fading, the channel gain $\left|\mathbf{h}_{11}\right|^{2}$ follows a Gamma distribution with parameters $k$ and $\theta$ [15]. Then, our proof is completed by deriving (18) based on the variable change $H_{11}=10 \log _{10}\left|\mathbf{h}_{11}\right|^{2}$ Details are omitted for brevity.

\section{The SIR Analysis}

From (10), we can approximate the UL SIR in $\mathrm{dB}$ by

$$
Z^{\mathrm{dB}} \approx X_{1}-Q \triangleq \hat{Z}^{\mathrm{dB}} \text {. }
$$

We derive the approximate distribution of $Z^{\mathrm{dB}}$ in Theorem 4.

Theorem 4. The approximate $C D F$ of $Z^{d B}$ is derived as $F_{Z^{d B}}(z) \approx F_{\hat{Z}^{d B}}(z)$

$$
=\frac{\lambda}{\sqrt{\pi}} \sum_{m=1}^{M_{0}} w_{m} \Phi^{\lambda-1}\left(\sqrt{2} a_{m}\right) F_{X_{1}}\left(z+\sqrt{2} \sigma_{Q} a_{m}+\mu_{Q}\right),
$$

where $M_{0},\left\{w_{m}\right\}$ and $\left\{a_{m}\right\}$ have the same definition as those in Theorem 1.

Proof: From (19), the approximate CDF of $Z^{\mathrm{dB}}$ can be derived as

$$
\begin{aligned}
& F_{Z^{\mathrm{dB}}}(z) \approx F_{\hat{Z}^{\mathrm{dB}}}(z) \\
& =\operatorname{Pr}\left[X_{1}-Q \leq z\right] \\
& =\operatorname{Pr}\left[X_{1} \leq z+Q\right] \\
& +\infty \\
& =\int_{-\infty}^{+\infty} F_{X_{1}}(z+q) f_{Q}(q) d q \\
& \stackrel{(a)}{\approx} \int_{-\infty}^{+\infty} F_{\hat{X}_{1}}(z+q) \lambda \Phi^{\lambda-1}\left(\frac{q-\mu_{Q}}{\sigma_{Q}}\right) \frac{1}{\sqrt{2 \pi \sigma_{Q}^{2}}} \exp \left\{-\frac{\left(q-\mu_{Q}\right)^{2}}{2 \sigma_{Q}^{2}}\right\} d q
\end{aligned}
$$$$
\stackrel{(b)}{=} \frac{1}{\sqrt{\pi}} \int_{-\infty}^{+\infty} F_{\hat{X}_{1}}\left(z+\sqrt{2} \sigma_{Q} y+\mu_{Q}\right) \lambda \Phi^{\lambda-1}(\sqrt{2} y) \exp \left(-y^{2}\right) d y
$$$$
\stackrel{(c)}{=} \frac{1}{\sqrt{\pi}} \sum_{m=1}^{M_{0}} w_{m} F_{\hat{X}_{1}}\left(z+\sqrt{2} \sigma_{Q} a_{m}+\mu_{Q}\right) \lambda \Phi^{\lambda-1}\left(\sqrt{2} a_{m}\right)+R_{M_{0}}
$$$$
\stackrel{(d)}{\approx} \frac{\lambda}{\sqrt{\pi}} \sum_{m=1}^{M_{0}} w_{m} \Phi^{\lambda-1}\left(\sqrt{2} a_{m}\right) F_{\hat{X}_{1}}\left(z+\sqrt{2} \sigma_{Q} a_{m}+\mu_{Q}\right),
$$

where the step (a) of (21) is calculated using Theorem 1 and (8), and the step (b) of (21) is computed using the variable change $q=\sqrt{2} \sigma_{Q} y+\mu_{Q}$. Moreover, the step (c) of (21) is derived using the Gauss-Hermite numerical integration [12]. Finally, the step (d) of (21) is obtained by dropping $R_{M_{0}}$. Our proof is thus completed by comparing (20) and (21).

In case of Rayleigh fading [15], we propose Corollary 5 to compute the approximate expression of $F_{Z^{\mathrm{dB}}}(z)$.

Corollary 5. In case of Rayleigh fading, the approximate CDF of $Z^{d B}$ can be computed by (20), where (17) is plugged into (14) to obtain $F_{\hat{X}_{1}}(x)$ in (20).

Proof: The proof is completed by applying Corollary 2 to Theorems 1 and 4. Details are omitted for brevity.

In case of Nakagami fading [15], we propose Corollary 6 to compute the approximate expression of $F_{Z^{\mathrm{dB}}}(z)$.

Corollary 6. In case of Nakagami fading, the approximate $C D F$ of $Z^{d B}$ can be computed by (20), where (18) is plugged into (14) to obtain $F_{\hat{X}_{1}}(x)$ in (20).

Proof: The proof is completed by applying Corollary 3 to Theorems 1 and 4. Details are omitted for brevity.

\section{MACROSCOPIC UPGRAdE OF THE DNA-GA ANALYSIS}

With Theorem 4, we have crafted a powerful microscopic analysis tool based on the proposed DNA-GA analysis that can deal with a wide range of network assumptions and system parameters. In this section, we further investigate two approaches for upgrading the DNA-GA analysis from a microscopic analysis tool to a macroscopic one, putting DNAGA in the same league as, e.g., stochastic geometry.

\section{A. The Semi-Analytical Approach}

The microscopic and the macroscopic analyses are closely related to each other. The average performance of many microscopic analyses conducted over a large number of random BS deployments converges to the performance of the macroscopic analysis, given that the examined realizations of the deterministic BS deployments follow the BS deployment assumption used in the macroscopic analysis. Therefore, we can directly average the performance results obtained by applying DNA-GA, i.e., Theorem 4, over a large number of random BS deployments to obtain the performance results of the macroscopic analysis.

\section{B. The Analytical Approach}

Instead of conducting DNA-GA, i.e., Theorem 4, over many $\mathrm{BS}$ deployments and averaging all the results together to obtain the results of the macroscopic analysis, we can construct an idealistic BS deployment on a hexagonal lattice with the equivalent BS density, and perform a single DNA-GA analysis on such BS deployment to extract an upper-bound of the SIR performance of the macroscopic analysis. The hexagonal lattice leads to an upper-bound performance because BSs are evenly distributed in the scenario and thus very strong interference due to close proximity is avoided $[3,4]$. 


\section{Simulation And Discussion}

In this section, we conduct simulations to validate the proposed DNA-GA analysis, using both the semi-analytical and the analytical approaches. For the semi-analytical approach, to obtain the results of the macroscopic analysis, we average the results given by Theorem 4 over 1000 random BS deployments. For each BS deployment, 10,000 random experiments are conducted to go through the randomness of UE positions. And for each BS deployment and each UE placement, another 10,000 random experiments are conducted to go through the randomness of shadow fading and multi-path fading. For the analytical approach, only one BS deployment in a hexagonal lattice is examined. $M_{0}$ is set to 30 for the computation in the DNA-GA analysis to ensure a good accuracy of the results [12].

With regard to the scenario and parameters, 3rd Generation Partnership Project (3GPP) recommendations have been considered [14]. For the semi-analytical approach, 19 dummy macrocell sites are deployed with a $0.5 \mathrm{~km}$ inter-site distance to guide the small cell deployment. Each macrocell site has the shape of a hexagon, and is equally divided into 3 macrocells. Each macrocell contains 4 randomly deployed small cells, resulting in $19 \times 3 \times 4=228$ small cells with a density around 55.43 cells $/ \mathrm{km}^{2}$. For the analytical approach, the 228 small cells are located in a hexagonal lattice with the same cell density. In both cases, each small cell has a coverage radius of $0.04 \mathrm{~km}$, and the minimum inter-BS distance and the minimum BS-to-UE distance are $0.04 \mathrm{~km}$, and $0.01 \mathrm{~km}$, respectively. Moreover, according to [14], $A=145.4, \alpha=3.75, P_{0}=-76$ $\mathrm{dBm}, \eta=0.8$, and $\sigma_{S}=10 \mathrm{~dB}$.

Fig. 2 illustrates an example of a random BS deployment according to [14], where small cell BSs are represented by $\mathrm{x}$ markers, while the coverage areas of dummy macrocells and small cells are marked by dashed and solid lines, respectively. UEs are randomly distributed in the mentioned small cell coverage areas, and it is important to note that although some small cell coverage areas are disk-shaped, the coverage areas of most small cells are of irregular shape due to overlapping.

For brevity, in the following subsections, we omit the detailed investigation on the interference analysis and the signal power analysis, and directly present SIR results given by the DNA-GA analysis and the simulation.

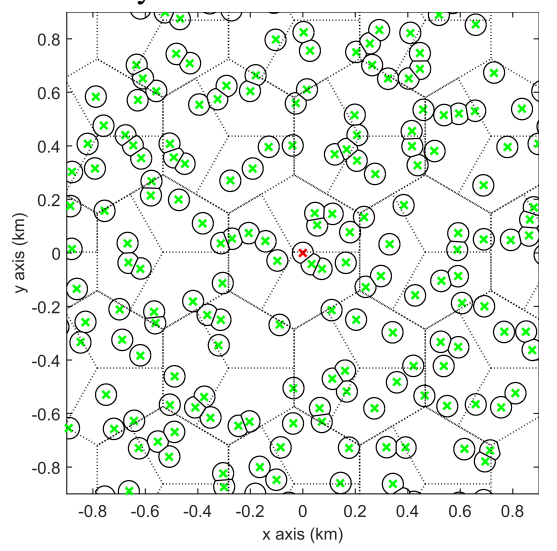

Fig. 2. Illustration of a 3GPP SCN deployment [14].

\section{A. Validation of the DNA-GA Analysis}

In this subsection, we validate the accuracy of the DNA-GA analysis in terms of the SIR performance when assuming two cases for UE distribution and multi-path fading, i.e.,

- Case 1: Uniform UE distribution + Rayleigh fading

- Case 2: Non-uniform UE distribution + Nakagami fading

For Case 1, we obtain the SIR results through the DNA-GA analysis using Theorem 4 and Corollary 5, while for Case 2, we invoke Theorem 4 and Corollary 6 .

When considering a non-uniform UE distribution, we assume that $f_{Z_{b}}(z)=\frac{W}{\rho}, z \in R_{b}$, where $\rho$ is the radial coordinate of $z$ in the polar coordinate system, the origin of which is placed at the position of the BS of $C_{b}$ and $W$ is a normalization constant to make $\int_{R_{b}} f_{Z_{b}}(z) d z=1$. In the resulting non-uniform UE distribution, UEs are more likely to locate in the close vicinity of the BS of $C_{b}$ than at the cell-edge ${ }^{2}$. When considering Nakagami fading, we assume that $k=10$ and $\theta=0.1$, which corresponds to a multi-path fading with a strong line-of-sight (LoS) component [15].

For both cases, the UL SIR performance is evaluated using the simulation and the semi-analytical approach discussed in Subsection IV-A. Moreover, the upper bound of the UL SIR is also investigated using the simulation and the analytical approach discussed in Subsection IV-B based on a BS deployment in a hexagonal lattice. The results are shown in Fig. 3.

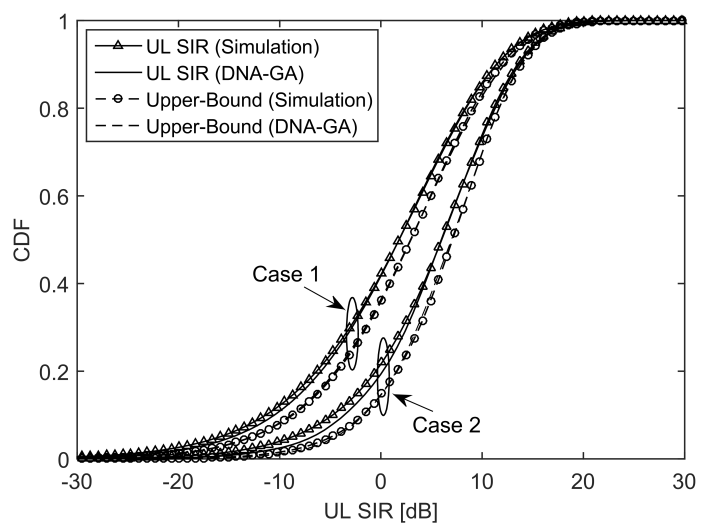

Fig. 3. UL SIR in dB (DNA-GA vs. Simulation).

As can be seen from Fig. 3, the SIR results of the proposed DNA-GA analysis match those of the simulation very well, particularly in the head portion. In the semi-analytical approach, the maximum deviation between the CDFs obtained by the DNA-GA analysis and the simulation for both investigated cases are around $1.0 \sim 1.7$ percentile. In the analytical approach, the fitness becomes even better, i.e., the maximum deviation for both cases is within 0.6 percentile.

More importantly, for both cases, the upper-bound SIR performance given by the analytical approach is shown to be within $2.0 \sim 2.5 \mathrm{~dB}$ from the exact performance, indicating its usefulness in characterizing the network performance with

\footnotetext{
${ }^{2}$ Note that the considered $f_{Z_{b}}(z)$ is just an example of the non-uniformly distributed UEs in $R_{b}$, which reflects a reasonable network planning, where small cell BSs have been deployed at the center of UE clusters. Other forms of $f_{Z_{b}}(z)$ can be considered in our DNA-GA analysis as well.
} 
low-complexity computation. To take Case 1 of DNA-GA as an example, the numerical results to be plugged into Theorem 4 for the hexagonal BS deployment are $\mu_{G_{1}}=-93.07$, $\sigma_{G_{1}}^{2}=5.97, \lambda=202.66, \mu_{Q}=-137.71$ and $\sigma_{Q}^{2}=212.04$.

Finally, note that the SIR of Case 2 outperforms that of Case 1, mainly because UEs tend to stay closer to their serving BSs in Case 2 as discussed above, leading to a larger signal power and a lower interference power.

\section{B. Comparison Between DNA-GA and Stochastic Geometry}

In this section, we compare the UL SIR results of the DNAGA analysis (Case 1) and those of the stochastic geometry analysis in Fig. 4, with the same average cell density of 55.43 cells $/ \mathrm{km}^{2}$ and the same assumption of Rayleigh fading ${ }^{3}$.

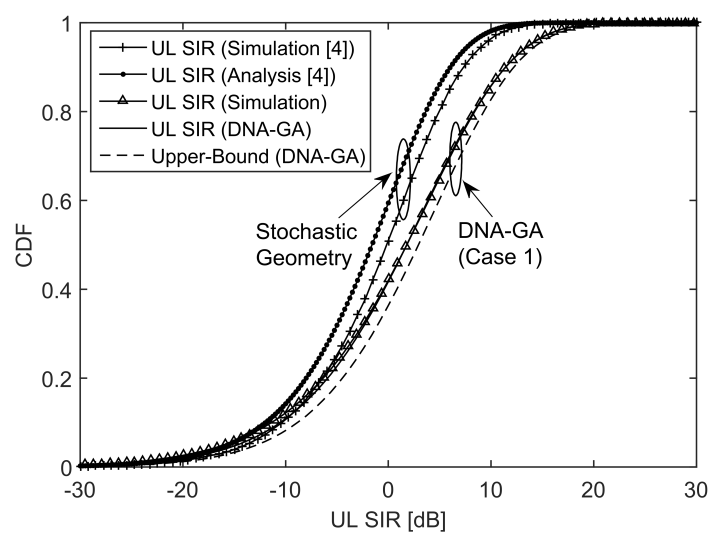

Fig. 4. UL SIR in dB (DNA-GA vs. Stochastic Geometry [4])

In Fig. 4, a few interesting aspects are noteworthy. First, both our analysis and the analysis in [4] are only able to give approximate results. However, the approximation error of our DNA-GA analysis is shown to be smaller than that of [4].

Second, there is a significant performance gap between our DNA-GA analysis and the stochastic geometry analysis in [4]. This is because (i) Our DNA-GA analysis considers the shadow fading on top of the multi-path fading, which leads to a large variance of SIR, while the analysis in [4] does not, which gives a small SIR variance; and (ii) The DNA-GA analysis studies the hotspot SCN scenario recommended by the 3GPP [14], where UEs are deployed closer to the serving BSs than those in Voronoi cells considered in [4].

Third, the purpose of Fig. 4 is not to reproduce the results in [4] based on Voronoi cells, but to analytically investigate a more practical 3GPP network scenario. If the shadow fading is required to be ignored, albeit impractical, the Gamma approximation of the aggregate interference [10] could be invoked to make our approach of analysis still valid. Besides, the DNA-GA analysis can also handle the case where the cell coverage areas are constructed as Voronoi cells [4]. However, to do so, it would be more practical to consider an alternative

\footnotetext{
${ }^{3}$ Note that the stochastic geometry analysis in [4] poses some assumptions on the model for the sake of tractability, e.g., no shadow fading, Rayleigh fading only, homogeneous Poisson distribution of both UEs and BSs in the entire scenario. In contrast, the DNA-GA analysis does not need such assumptions and works with a more realistic model, considering the hotspot SCN scenario [14] shown in Fig. 2 and discussed in Remark 2 of Section II.
}

UE association strategy (UAS), where each UE is connected to the BS with the smallest path loss plus shadow fading. Note that such UAS will blur the boundaries of Voronoi cells, because each UE is no longer always connected to its closest $\mathrm{BS}$, making the analysis more intricate and realistic.

Finally, note that a three-fold integral computation is needed in [4] to compute the results, while no integration is required in Theorem 4 of the DNA-GA analysis. However, many BS deployments are needed in the semi-analytical approach of the DNA-GA analysis, while only one in the analytical approach.

\section{CONCLUSION}

We proposed a new approach of network performance analysis, which unifies the microscopic and the macroscopic analyses within a single framework. Compared with stochastic geometry, our DNA-GA analysis considers shadow fading, general UE distribution and any type of multi-path fading, as well as any shape and/or size of cell coverage areas.

\section{REFERENCES}

[1] 3GPP, "TR 36.872, Small cell enhancements for E-UTRA and EUTRAN - Physical layer aspects," Dec. 2013.

[2] D. López Pérez, M. Ding, H. Claussen, and A. H. Jafari, “Towards $1 \mathrm{Gbps} / \mathrm{UE}$ in cellular systems: Understanding ultra-dense small cell deployments," IEEE Commun. Surveys and Tutorials, vol. 17, no. 4, pp. 2078-2101, Jun. 2015.

[3] J. G. Andrews, F. Baccelli, and R. K. Ganti, "A tractable approach to coverage and rate in cellular networks," IEEE Trans. on Commun., vol. 59, no. 11, pp. 3122-3134, Nov. 2011.

[4] T. D. Novlan, H. S. Dhillon and J. G. Andrews, "Analytical modeling of uplink cellular networks," IEEE Trans. on Wireless Commun., vol. 12, no. 6, pp. 2669-2679, Jun. 2013.

[5] Y. Zhu, J. Xu, Z. Hu, J. Wang and Y. Yang, "Distribution of uplink inter-cell interference in OFDMA networks with power control," IEEE ICC 2014, Sydney, Australia, pp. 5729-5734, Jun. 2014.

[6] J. He, Z. Tang, H. Chen, and W. Cheng, "Statistical model of OFDMA cellular networks uplink interference using lognormal distribution,' IEEE Wireless Commun. Letters, vol. 2, no. 5, pp. 575-578, Oct. 2013.

[7] M. Ding, D. López-Pérez, G. Mao, and Z. Lin, "Approximation of uplink inter-cell interference in FDMA small cell networks," to appear in IEEE Globecom 2015, arXiv: 1505.01924 [cs.NI], May 2015.

[8] M. Ding, D. López-Pérez, G. Mao, and Z. Lin, "Microscopic analysis of the uplink interference in FDMA small cell networks," to appear in IEEE Trans. on Wireless Commun., arXiv:1508.02808, Aug. 2015.

[9] M. Ding, D. López Pérez, A. V. Vasilakos, and W. Chen, "Dynamic TDD transmissions in homogeneous small cell networks," IEEE ICC 2014, Sydney, Australia, pp. 616-621, Jun. 2014.

[10] M. Ding, D. López Pérez, A. V. Vasilakos, and W. Chen, "Analysis on the SINR performance of dynamic TDD in homogeneous small cell networks," IEEE Globecom 2014, pp. 1552-1558, Dec. 2014.

[11] F. J. Massey Jr. "The Kolmogorov-Smirnov test for goodness of fit," Journal of the American Statistical Association, vol. 46, no. 253, pp. 68-78, 1951.

[12] M. Abramowitz and I. Stegun, Handbook of mathematical functions with formulas, graphs, and mathematical tables (Nineth Ed.), Dover, 1972.

[13] WiMax Forum, "WiMAX and the IEEE 802.16m Air Interface Standard," Apr. 2010.

[14] 3GPP, "TR 36.828 (V11.0.0): Further enhancements to LTE Time Division Duplex (TDD) for Downlink-Uplink (DL-UL) interference management and traffic adaptation," Jun. 2012.

[15] J. Proakis, Digital Communications (Third Ed.), New York: McGrawHill, 1995.

[16] Z. Liu, J. Almhana, and R. McGorman, "Approximating lognormal sum distributions with power lognormal distributions," IEEE Trans. on Vehicular Tech., vol. 57, no. 4, pp. 2611-2617, Jul. 2008.

[17] S. S. Szyszkowicz and H. Yanikomeroglu, "Fitting the modified-powerlognormal to the sum of independent lognormals distribution," IEEE Globecom 2009, pp. 1-6, Nov. 2009. 